\title{
On the integrability of a system describing the stationary solutions in Bose-Fermi mixtures
}

\author{
Ognyan Christov ${ }^{\mathrm{a}, *}$, Georgi Georgiev ${ }^{\mathrm{b}}$ \\ ${ }^{a}$ Faculty of Mathematics and Informatics, Sofia University, 1164 Sofia, Bulgaria \\ ${ }^{b}$ Department of Mathematics and Informatics, University of Transport, Sofia, Bulgaria
}

\begin{abstract}
We study the integrability of a Hamiltonian system describing the stationary solutions in Bose-Fermi mixtures in one dimensional optical lattices. We prove that the system is integrable in the Liouville sense only when it is separable in three generic cases. The proof is based on the Differential Galois approach and the Ziglin-Morales-Ramis method.
\end{abstract}

Keywords: Bose-Fermi mixtures, Liouville integrability, Differential Galois groups, Ziglin-Morales-Ramis approach

\section{Introduction}

In this paper we study the integrability of the system that comes from the time dependent mean field equations of Bose-Fermi mixture (BFM) in one dimensional optical lattices. The interest in BFM arises after the discovery of Bose-Einstein Condensates (BEC) in 1995 and the desire to understand strongly interacting and strongly correlated systems, with applications in solid state physics, nuclear physics, astrophysics, quantum computing and nanotechnologies. For more detailed physical background of BFM we refer to $[8,19,4,5,9]$ and the literature therein.

At mean field approximation we consider the following $N_{f}+1$ coupled nonlinear Schrödinger equations

$$
\begin{aligned}
& i \hbar \frac{\partial \Psi^{b}}{\partial t} \quad+\frac{1}{2 m_{\mathrm{B}}} \frac{\partial^{2} \Psi^{b}}{\partial x^{2}}-V \Psi^{b}-g_{\mathrm{BB}}\left|\Psi^{b}\right|^{2} \Psi^{b}-g_{\mathrm{BF}} \rho_{f} \Psi^{b}=0, \\
& i \hbar \frac{\partial \Psi_{j}^{f}}{\partial t}+\frac{1}{2 m_{\mathrm{F}}} \frac{\partial^{2} \Psi_{j}^{f}}{\partial x^{2}}-V \Psi_{j}^{f}-g_{\mathrm{BF}}\left|\Psi^{b}\right|^{2} \Psi_{j}^{f}=0, \quad j=1, \ldots, N_{f}
\end{aligned}
$$

\footnotetext{
${ }^{*}$ Corresponding author
} 
where the wavefunctions $\Psi_{j}^{f}$ describe each of $N_{f}$ fermions and $\Psi^{b}$ is the wavefunction for the bosonic component, $\rho_{f}=\sum_{i=1}^{N_{f}}\left|\Psi_{i}^{f}\right|^{2}$ and $g_{\mathrm{BB}}, g_{\mathrm{BF}}, m_{\mathrm{F}}, m_{\mathrm{B}}$ are certain physical constants. In particular, $g_{\mathrm{BB}}$ and $g_{\mathrm{BF}}$ are related with the s-wave collisions for bosonboson and boson-fermion interactions, respectively. The potential $V$ is usually of the form $V=V_{0} s n^{2}(\alpha x, \kappa)$, where $s n(\alpha x, \kappa)$ is the Jacobi elliptic sine function. In this paper we take $V_{0}=0$ as in [4].

We are interested in the stationary solutions to the system (1.1), (1.2) of the kind

$$
\begin{aligned}
& \Psi^{b}(x, t)=q_{0}(x) \exp \left(-i \frac{\omega_{0}}{\hbar} t+i \Theta_{0}(x)+i \kappa_{0}\right), \\
& \Psi_{j}^{f}(x, t)=q_{j}(x) \exp \left(-i \frac{\omega_{j}}{\hbar} t+i \Theta_{j}(x)+i \kappa_{0, j}\right), \quad j=1, \ldots, N_{f},
\end{aligned}
$$

where $\kappa_{0}, \kappa_{0, j}$ are constant phases, $q_{0}, q_{j}$ and $\Theta_{0}, \Theta_{j}$ are real-valued functions related by

$$
\Theta_{0}(x)=C_{0} \int_{0}^{x} \frac{d x^{\prime}}{q_{0}^{2}\left(x^{\prime}\right)}, \quad \Theta_{j}(x)=C_{j} \int_{0}^{x} \frac{d x^{\prime}}{q_{j}^{2}\left(x^{\prime}\right)}, \quad j=1, \ldots, N_{f}
$$

$C_{0}, C_{j}$, being constants of integration. After substituting (1.3), (1.4) in the equations (1.1), (1.2) and separating the real and imaginary part we get

$$
\begin{aligned}
& \frac{1}{2 m_{\mathrm{B}}} q_{0}^{3} q_{0 x x}-g_{\mathrm{BB}} q_{0}^{6}-g_{\mathrm{BF}}\left(\sum_{i=1}^{N_{f}} q_{i}^{2}\right) q_{0}^{4}+\omega_{0} q_{0}^{4}=\frac{C_{0}^{2}}{2 m_{\mathrm{B}}}, \\
& \frac{1}{2 m_{\mathrm{F}}} q_{j}^{3} q_{j x x}-g_{\mathrm{BF}} q_{0}^{2} q_{j}^{4}+\omega_{j} q_{j}^{4}=\frac{C_{j}^{2}}{2 m_{\mathrm{F}}}, \quad j=1, \ldots, N_{f} .
\end{aligned}
$$

Kostov et al. [9] have found plenty of particular (quasiperiodic, periodic and soliton) solutions to the system (1.6) and therefore, stationary solutions to the system (1.1), (1.2). It is natural to ask whether we can obtain more, that is, for what set of the parameters the system (1.6) has enough first integrals to be integrable. Note that when $g_{\mathrm{BF}}=0$ the equations separate, i.e., the system is solvable.

Before giving our main result let first get rid of the inessential (for integrability) parameters. In what follows we assume that the parameters $\omega_{0}, \omega_{j}, m_{\mathrm{F}}, m_{\mathrm{B}}, g_{\mathrm{BB}}$ are positive since they have an origin from physics, and $C_{0}, C_{j}, g_{\mathrm{BF}}$ are arbitrary real parameters. We put $q_{0}=\beta \tilde{q}_{0}, q_{j}=\alpha \tilde{q}_{j}, x=\gamma \tilde{x}$. Then we choose $\alpha=\sqrt{m_{\mathrm{F}}}, \beta=$ $\sqrt{m_{\mathrm{B}}}, \gamma=1 /\left(m_{\mathrm{B}} \sqrt{g_{\mathrm{BB}}}\right), g_{\mathrm{BB}} \neq 0$. Denoting $\tilde{g}_{\mathrm{BF}}=g_{\mathrm{BF}} \alpha^{2} \gamma^{2} m_{\mathrm{B}}, \tilde{\omega}_{0}=\omega_{0} \gamma^{2} m_{\mathrm{B}}, \tilde{\omega}_{j}=$ 
$\omega_{j} \gamma^{2} m_{\mathrm{F}}, \tilde{C}_{j}^{2}=C_{j}^{2} \gamma^{2} / \alpha^{4}, \tilde{C}_{0}^{2}=C_{0}^{2} \gamma^{2} / \beta^{4}$ we reach

$$
\begin{gathered}
\frac{1}{2} \frac{d^{2} \tilde{q}_{0}}{d \tilde{x}^{2}}-\tilde{q}_{0}^{3}-\tilde{g}_{\mathrm{BF}}\left(\sum_{i=1}^{N_{f}} \tilde{q}_{i}^{2}\right) \tilde{q}_{0}+\tilde{\omega}_{0} \tilde{q}_{0}=\frac{\tilde{C}_{0}^{2}}{2 \tilde{q}_{0}^{3}}, \\
\frac{1}{2} \frac{d^{2} \tilde{q}_{j}}{d \tilde{x}^{2}}-\tilde{g}_{\mathrm{BF}} \tilde{q}_{0}^{2} \tilde{q}_{j}+\tilde{\omega}_{j} \tilde{q}_{j}=\frac{\tilde{C}_{j}^{2}}{2 \tilde{q}_{j}^{3}}, \quad j=1, \ldots, N_{f} .
\end{gathered}
$$

To simplify notations we skip the tildes, write $t$ instead of $x$ and denote $p_{j}=\dot{q}_{j}, j=$ $0, \ldots, N_{f},(.=d / d t)$. Then the system (1.7) can be presented as a Hamiltonian system with the Hamiltonian

$$
H=\frac{p_{0}^{2}}{2}+\frac{1}{2} \sum_{1}^{N_{f}} p_{j}^{2}+\omega_{0} q_{0}^{2}+\sum_{1}^{N_{f}} \omega_{j} q_{j}^{2}-g_{\mathrm{BF}} q_{0}^{2} \sum_{1}^{N_{f}} q_{j}^{2}-\frac{q_{0}^{4}}{2}+\frac{C_{0}^{2}}{2 q_{0}^{2}}+\frac{1}{2} \sum_{1}^{N_{f}} \frac{C_{j}^{2}}{q_{j}^{2}}
$$

For the Hamiltonian system with the Hamiltonian (1.8) we consider the cases:

1) $C_{0}=0, C_{j} \neq 0, \sum C_{j} \neq 0, \omega_{j}=\omega^{2} / 2, j=1, \ldots, N_{f}$;

2) $C_{0} \neq 0, C_{j}=0, j=1, \ldots, N_{f}, g_{\mathrm{BF}}=n(n+1) / 2, n \notin \mathbb{Z}$;

3) $C_{0} \neq 0, C_{1} \neq 0, N_{f}=1, g_{\mathrm{BF}}$ sufficiently small.

Our result is the following:

Theorem 1. For the cases given above, the Hamiltonian system corresponding to (1.8) is non-integrable in the Liouville sense unless $g_{\mathrm{BF}}=0$.

In other words, the Hamiltonian system under consideration is integrable only when it is separable.

The proof of the above result is based on the Differential Galois approach and the Ziglin-Morales-Ramis method. This method has been applied for the studying the integrability to a number of Hamiltonian systems, in particular systems with homogeneous potentials, see $[12,13,14,17]$. The classification of all integrable two degrees of freedom systems with polynomial potentials of degree 3 is obtained in [10]. The above mentioned approach is used in [1] for obtaining non-integrability results for some two degrees of freedom Hamiltonians with rational potentials. Note that the system in this paper is not of that kind.

For the natural Hamiltonian systems with two degrees of freedom, similar to (1.8)

$$
H=\frac{p_{1}^{2}+p_{2}^{2}}{2}+U\left(q_{1}, q_{2}\right)
$$


there is an integrable generalization of Garnier's system found by Wojciechowski [21], namely

$$
U=A q_{1}^{2}+B q_{2}^{2}+\left(q_{1}^{2}+q_{2}^{2}\right)^{2}+\frac{C}{q_{1}^{2}}+\frac{D}{q_{2}^{2}},
$$

with a rational first integral depending on $A, B, C, D$ (see also [16]). Note that in the system under consideration, the symmetry is lost, so it is natural to expect integrability only in the separable case.

Let us turn to the case 2 above : $C_{0} \neq 0, C_{j}=0, j=1, \ldots, N_{f}$ when $g_{\mathrm{BF}}=$ $n(n+1) / 2, n \in \mathbb{Z}$. We formulate the following

Conjecture. The Hamiltonian system corresponding to (1.8) when $C_{0} \neq 0, C_{j}=$ $0, j=1, \ldots, N_{f}$ and $g_{\mathrm{BF}}=n(n+1) / 2, n \in \mathbb{Z}$ is non-integrable in the Liouville sense unless $g_{\mathrm{BF}}=0$.

This statement is formulated in that way because we have checked it only for $n=1$ and $n=2$. Nevertheless, we think that the system (1.8) is also non-integrable for arbitrary integer $n>2$.

The paper is organized as follows. In the next section we recall some facts about the Differential Galois groups and the Morales-Ramis method which we use. Then, Section 3 is devoted to the proof of Theorem 1. Some comments are made in Section 4. We also give the proofs that the corresponding system is indeed non-integrable for the cases $n=1$ and $n=2$ in the above Conjecture there.

\section{Differential Galois Theory and Integrability}

Here we summarize some notions and results related to the Ziglin-Morales-RamisSimó theory.

A differential field is a field with derivation $\partial=^{\prime}$, i.e. an additive mapping satisfying Leibnitz rule. A differential automorphism of $K$ is an automorphism commuting with the derivation.

Consider a linear system

$$
\dot{x}=A(t) x, \quad x \in \mathbb{C}^{n}
$$

with $t$ defined on some Riemann surface. Denote the coefficient field in (2.1) by $K$. Let $x_{i j}$ be the elements of the fundamental matrix $X(t)$. Let $L\left(x_{i j}\right)$ be the extension of $K$ generated by $K$ and $x_{i j}$ - a differential field. This extension is called PicardVessiot extension. Similarly to classical Galois Theory we define the Galois group $G:=\operatorname{Gal}_{K}(L)=\operatorname{Gal}(L / K)$ to be the group of all differential automorphisms of $L$ 
leaving the elements of $K$ fixed. The Galois group is, in fact, an algebraic group. It has a unique connected component $G^{0}$ which contains the identity and which is a normal subgroup of finite index. The Galois group $G$ can be represented as an algebraic linear subgroup of $G L(n, \mathbb{C})$ by

$$
\sigma(X(t))=X(t) R_{\sigma}
$$

where $\sigma \in G$ and $R_{\sigma} \in G L(n, \mathbb{C})$ (see e.g. [20]).

Consider now a Hamiltonian system

$$
\dot{x}=X_{H}(x), \quad t \in \mathbb{C}, \quad x \in M
$$

corresponding to an analytic Hamiltonian $H$, defined on the complex $2 n$-dimensional manifold $M$. Suppose the system $(2.2)$ has a non-equilibrium solution $\Psi(t)$. Denote by $\Gamma$ its phase curve. We can write the equation in variation (VE) along this solution

$$
\dot{\xi}=D X_{H}(\Psi(t)) \xi, \quad \xi \in T_{\Gamma} M .
$$

Further, using the integral $d H$ we can reduce the variational equation. Consider the normal bundle of $\Gamma, F:=T_{\Gamma} M / T M$ and let $\pi: T_{\Gamma} M \rightarrow F$ be the natural projection. The equation (2.3) induces an equation on $F$

$$
\dot{\eta}=\pi_{*}\left(D X_{H}(\Psi(t))\left(\pi^{-1} \eta\right), \quad \eta \in F .\right.
$$

which is called the normal variational equation (NVE).

The solutions of (2.3) define an extension $L_{1}$ of the coefficient field $K$ of (VE). This naturally defines a differential Galois group $G=\operatorname{Gal}\left(L_{1} / K\right)$. Then, the following result has established

Theorem 2. (Morales-Ruiz-Ramis [12]) Suppose that a Hamiltonian system has $n$ meromorphic first integrals in involution. Then the identity component $G^{0}$ of the Galois group $G=\operatorname{Gal}\left(L_{1} / K\right)$ is abelian.

Once it is proven, that $G^{0}$ is not abelian, the respective Hamiltonian system is non-integrable in the Liouville sense. Note that the fact that $G^{0}$ is abelian doesn't imply necessarily integrability of the Hamiltonian system. Thus, one needs other obstructions to the integrability. A method based on the higher variational equations has been introduced in [12] and the previous Theorem has been extended in [13]. Before formulating this result let us give an idea of higher variational equations. For the system $(2.2)$ with a particular solution $\Psi(t)$ we put

$$
x=\Psi(t)+\varepsilon \xi^{(1)}+\varepsilon^{2} \xi^{(2)}+\ldots+\varepsilon^{k} \xi^{(k)}+\ldots,
$$


where $\varepsilon$ is a formal small parameter. Substituting the above expression into Eq. (2.2) and comparing terms with the same order in $\varepsilon$ we obtain the following chain of linear non-homogeneous equations

$$
\dot{\xi}^{(k)}=A(t) \xi^{(k)}+f_{k}\left(\xi^{(1)}, \ldots, \xi^{(k-1)}\right), \quad k=1,2, \ldots,
$$

where $A(t)=D X_{H}(\Psi(t))$ and $f_{1} \equiv 0$. The equation (2.6) is called k-th variational equation $\left(\mathrm{VE}_{k}\right)$. Let $X(t)$ be the fundamental matrix of $\left(\mathrm{VE}_{1}\right)$

$$
\dot{X}=A(t) X
$$

Then the solutions of $\left(\mathrm{VE}_{k}\right), k>1$ can be found by

$$
\xi^{(k)}=X(t) c(t)
$$

where $c(t)$ is a solution of

$$
\dot{c}=X^{-1}(t) f_{k}
$$

Although $\left(\mathrm{VE}_{k}\right)$ are not actually homogeneous equations, they can be put in that frame, and therefore, one can define successive extensions $K \subset L_{1} \subset L_{2} \subset \ldots \subset L_{k}$, where $L_{k}$ is the extension obtained by adjoining the solutions of $\left(\mathrm{VE}_{k}\right)$. Correspondingly one can define the differential Galois groups $\operatorname{Gal}\left(L_{1} / K\right), \ldots, \operatorname{Gal}\left(L_{k} / K\right)$. The following result is proven in [13].

Theorem 3. If the Hamiltonian system (2.2) is integrable in Liouville sense then the identity component of every Galois group $\operatorname{Gal}\left(L_{k} / K\right)$ is abelian.

Note that we apply Theorem 3 in the situation when the identity component of the Galois group $\operatorname{Gal}\left(L_{1} / K\right)$ is abelian. This means that the first variational equation is solvable. Once we have the solution of $\left(\mathrm{VE}_{1}\right)$, then the solutions of $\left(\mathrm{VE}_{k}\right)$ can be found by the method of variations of constants as explained above. Hence, the differential Galois groups $\operatorname{Gal}\left(L_{k} / K\right)$ are solvable. One possible way to show that some of them is not commutative is to find a logarithmic term in the corresponding solution (see detailed descriptions and explanations in $[12,13,14]$ ).

Now we recall a perturbational technique which is still related to the Differential Galois approach. Let $M_{0}$ be a two-dimensional complex analytic symplectic manifold, $H_{0}(q, p)$ be a holomorphic Hamiltonian and $X_{H_{0}}$ be the corresponding Hamiltonian vector field. Assume that the system

$$
\dot{q}=H_{0, p}, \quad \dot{p}=-H_{0, q}
$$


has a hyperbolic equilibrium $\left(q_{0}, p_{0}\right)$. Then the system $(2.9)$ has a separatrix

$$
\Gamma_{0}:\left(q_{0}(t), p_{0}(t)\right), \lim _{t \rightarrow \infty} q_{0}(t)=q_{0}, \lim _{t \rightarrow \infty} p_{0}(t)=p_{0} .
$$

The functions $q_{0}(t), p_{0}(t)$ are meromorphic in $t \in \mathbb{C}$. Let

$$
H(q, p, t, \varepsilon)=H_{0}(q, p)+\varepsilon H_{1}(q, p, t)+\ldots
$$

be a meromorphic small (complex) perturbation of $H_{0}$ satisfying $H_{1}(q, p, t+\omega)=$ $H_{1}(q, p, t)$ with a period $\omega \in \mathbb{C}$. This function $H$ is defined over $M=M_{0} \times F_{\omega}, F_{\omega}=$ $\mathbb{C} / \omega \mathbb{Z}$. We can write the Hamiltonian system defined by $H(q, p, \varphi)$ over $M$ as

$$
\dot{q}=H_{p}, \quad \dot{p}=-H_{q}, \quad \dot{\varphi}=1, \quad(q, p, \varphi) \in M .
$$

When $\varepsilon=0$ the system (2.12) reduces to

$$
\dot{q}=H_{0, p}, \quad \dot{p}=-H_{0, q}, \quad \dot{\varphi}=1, \quad(q, p, \varphi) \in M .
$$

The unperturbed system (2.13) has a hyperbolic $\omega$-periodic orbit $\Pi_{0}:=\left(q_{0}, p_{0}, \varphi=t(\right.$ $\bmod \omega))$. It is well known that for small $|\varepsilon|$ the perturbed system (2.12) has also an $\omega$ periodic orbit $\Pi_{\varepsilon}:=\left(q(t, \varepsilon), p(t, \varepsilon), \varphi=t-t_{0}(\bmod \omega)\right)$, such that $(q(t, 0), p(t, 0))=$ $\left(q_{0}, p_{0}\right)$.

We define the (stable) complex separatrix $\Lambda_{\varepsilon}^{+}$of the system (2.12) as the set of integral curves of (2.12) asymptotic to $\Pi_{\varepsilon}$ as $t \rightarrow \infty$. For fixed $\varepsilon$, it is a twodimensional complex surface. This separatrix can have transverse self-intersections.

Remark 1. Recall that in the real case the separatrices can not have transverse self-intersections. Such intersections can occur between stable and unstable separatrices. For real Hamiltonian systems, the existence of such transverse orbits is considered as a source of chaotic behavior and is an obstruction to existence of an analytic first integral.

Ziglin [22] proved that for complex Hamiltonian systems, the existence of transverse self-intersections for separatrices is also an obstruction to the integrability.

The unperturbed separatrix is given by $\Lambda_{0}^{+}=\Gamma_{0} \times F_{\omega}$. It is foliated by the one-parameter family of integral curves

$$
\Gamma_{t_{0}}:\left(q_{0}(t), p_{0}(t), t-t_{0}\right),
$$

$t_{0} \in F_{\omega}$ being the parameter. Let $\gamma:[0,1] \rightarrow \mathbb{C}$ be a closed path in the complex plane with $\gamma(0)=\gamma(1) \in \mathbb{R} \subset \mathbb{C}$. The following function on $F_{\omega}$

$$
d\left(t_{0}\right):=\int_{\gamma}\left\{H_{0}, H_{1}\right\}\left(q_{0}(t), p_{0}(t), t-t_{0}\right) d t
$$


is usually called the Poincaré-Arnold-Melnikov integral. Here $\{$,$\} is the Poisson$ bracket. Then the following result is valid:

Theorem 4. (Ziglin) If the function $d\left(t_{0}\right)$ has a simple zero, then for sufficiently small $|\varepsilon| \neq 0$, the separatrix $\Lambda_{\varepsilon}^{+}$has a transversal self-intersection and the system (2.12) has no additional holomorphic first integral.

It appears that there is a relation between Theorem 2 and Theorem 4. MoralesRuiz [15] proved that, under certain assumptions, the Ziglin's condition about the Poincaré-Arnold-Melnikov integral can be interpreted by the fact that the Galois group of the perturbed variational equation along the integral curve $\Gamma_{0}$ is non-abelian. In other words, if the Poincaré-Arnold-Melnikov integral $d\left(t_{0}\right)$ is not identically zero, the differential Galois group of the perturbed variational equation is not abelian and the system is not integrable by means of meromorphic first integrals.

\section{Proof of Theorem 1}

In what follows we assume that $t, q_{0}(t), q_{j}(t)$ are complex quantities, but we keep the parameters real. The proof goes in the following lines. For the first two parts we find particular solutions. Then we study the variational equation (VE) along these solutions. The first part is the simplest, that is why we start with it. The variational equation (VE) is reduced to a particular case of the double confluent Heun equation, which differential Galois group is more or less known.

The second part needs more steps. The identity component of the Galois group of (VE) is not commutative except for some discrete values of $g_{\mathrm{BF}}$. By studying higher variational equations we find a logarithmic term in solutions of $\left(\mathrm{VE}_{3}\right)$ when $g_{\mathrm{BF}} \neq 0$, which implies non commutativity of the identity component of $\operatorname{Gal}\left(L_{3} / K\right)$ and hence, non-integrability of our Hamiltonian system.

For the third part we use a perturbational technique which is still related to the Differential Galois approach. We study the Poincaré-Arnold-Melnikov integral in order to show that a complex separatrix self-intersects.

\subsection{The case $C_{0}=0, C_{j} \neq 0$.}

In this case the Hamiltonian (1.8) becomes

$$
H=\frac{p_{0}^{2}}{2}+\frac{1}{2} \sum_{1}^{N_{f}} p_{j}^{2}+\omega_{0} q_{0}^{2}+\sum_{1}^{N_{f}} \omega_{j} q_{j}^{2}-g_{\mathrm{BF}} q_{0}^{2} \sum_{1}^{N_{f}} q_{j}^{2}-\frac{q_{0}^{4}}{2}+\frac{1}{2} \sum_{1}^{N_{f}} \frac{C_{j}^{2}}{q_{j}^{2}} .
$$


The equations corresponding to the Hamiltonian (3.1) are

$$
\begin{aligned}
& \dot{q_{0}}=p_{0}, \quad \dot{p_{0}}=-2 \omega_{0} q_{0}+2 q_{0}^{3}+2 g_{\mathrm{BF}} q_{0} \sum_{1}^{N_{f}} q_{j}{ }^{2}, \\
& \dot{q}_{j}=p_{j}, \quad \dot{p_{j}}=-2 \omega_{j} q_{j}+2 g_{\mathrm{BF}} q_{0}^{2} q_{j}+\frac{C_{j}^{2}}{q_{j}^{3}}, \quad j=1, \ldots, N_{f} .
\end{aligned}
$$

Proposition 1. The system (3.2) has a particular solution of the form

$$
\begin{gathered}
q_{0}=p_{0}=0, \\
q_{j}{ }^{2}=\frac{C_{j}}{\sqrt{2 \omega_{j}}} \sinh \left(2 i \sqrt{2 \omega_{j}} t\right), \quad p_{j}=\dot{q}_{j}, \quad j=1, \ldots, N_{f} .
\end{gathered}
$$

Proof. We put $q_{0}=p_{0}=0$ in (3.2). The general solution of the system with respect to $\left(q_{j}, p_{j}\right), j=1, \ldots, N_{f}$ is

$$
q_{j}^{2}=\frac{h_{j}}{2 \omega_{j}}+\sqrt{\frac{C_{j}^{2}}{2 \omega_{j}}-\frac{h_{j}^{2}}{4 \omega_{j}^{2}}} \sinh 2 i \sqrt{2 \omega_{j}}\left(t-t_{0}\right), \quad p_{j}=\dot{q}_{j}, \quad j=1, \ldots, N_{f},
$$

here $h_{j}$ are arbitrary constants. Then we set $h_{j}=0$ and $t_{0}=0$ to obtain our particular solution.

Denote the variations by $\xi_{0}=d q_{0}$ and $\eta_{0}=d p_{0}$. It is easy to see that the (NVE) are written in variables $\xi_{0}, \eta_{0}$, namely

$$
\dot{\xi}_{0}=\eta_{0}, \quad \dot{\eta}_{0}=\left[-2 \omega_{0}+2 g_{\mathrm{BF}} \sum_{1}^{N_{f}} q_{j}{ }^{2}\right] \xi_{0}
$$

We rewrite (3.5) as a second order equation

$$
\ddot{\xi}_{0}+\left[2 \omega_{0}-2 g_{\mathrm{BF}} \sum_{1}^{N_{f}} \frac{C_{j}}{\sqrt{2 \omega_{j}}} \sinh \left(2 i \sqrt{2 \omega_{j}} t\right)\right] \xi_{0}=0 .
$$

The study of the identity component of the Galois group of (3.6) is a difficult task. That is why we assume that all $\omega_{j}$ are equal. We put $\omega_{j}=\frac{\omega^{2}}{2}, j=1, \ldots, N_{f}$. Then we get a variant of the Mathieu equation

$$
\ddot{\xi}_{0}+\left[A_{1}+B_{1} \sinh (2 i \omega t)\right] \xi_{0}=0
$$


where

$$
A_{1}=2 \omega_{0}, \quad B_{1}=-\frac{2}{\omega} g_{\mathrm{BF}} \sum_{1}^{N_{f}} C_{j} .
$$

Since $C_{j}$ are constants of integration, we can always assume that $\sum C_{j} \neq 0$.

Next, by changing the independent variable $x=e^{2 i \omega t}$ we get an algebraic version of $(3.7)$

$$
\xi_{0}^{\prime \prime}+\frac{1}{x} \xi_{0}^{\prime}+\left[\frac{B}{x}+\frac{A}{x^{2}}-\frac{B}{x^{3}}\right] \xi_{0}=0,
$$

where $^{\prime}=\frac{d}{d x}, A=-\frac{A_{1}}{4 \omega^{2}}, B=-\frac{B_{1}}{8 \omega^{2}}$. It is obvious that when $B=0$ this equation becomes an Euler equation which is solvable. Further, we reduce (3.9) to the standard form by putting $y=\sqrt{x} \xi_{0}$,

$$
y^{\prime \prime}=r(x) y, \quad r(x)=-\frac{B}{x}-\frac{A+\frac{1}{4}}{x^{2}}+\frac{B}{x^{3}} .
$$

The equation (3.10) is a particular case of the double confluent Heun equation. For this equation the points 0 and $\infty$ are irregular singular ones and one natural way to study the Galois group is the Kovacic algorithm. This is done by A. Duval and M. Loday-Richaud in [6] p.237. We just apply their result which simply says that if $B \neq 0$ the Galois group of $(3.10)$ is $\operatorname{SL}(2, \mathbb{C})$. In our case

$$
B=\frac{g_{\mathrm{BF}}}{4 \omega^{3}} \sum_{1}^{N_{f}} C_{j}
$$

which means that under the assumption $\sum_{1}^{N_{f}} C_{j} \neq 0$

$$
B=0 \Leftrightarrow g_{\mathrm{BF}}=0
$$

that is, the identity component of the Galois group is noncommutative if $g_{\mathrm{BF}} \neq 0$. Therefore, by Theorem 2 the Hamiltonian system (3.1) is non-integrable unless $g_{\mathrm{BF}}=$ 0 . This finishes the proof of this part of Theorem 1 .

Remark 2. Let us note that in $[1,2,3]$ a systematic procedure is presented, called Hamiltonian Algebrization, which transforms second order linear differential equations with non-rational coefficients into differential equations with rational coefficients. As an example, the Mathieu equation is considered, see for instance section 2.1 in [1]. The conclusion is the same: the Mathieu equation is not integrable for $B \neq 0$. 


\subsection{The case $C_{0} \neq 0, C_{j}=0$.}

Let us first find a particular solution.

Proposition 2. The Hamiltonian system generated by the Hamiltonian (1.8) with $C_{j}=0$ has a particular solution in the form

$$
\bar{q}_{0}^{2}(t)=\frac{2}{3} \omega_{0}+\wp\left(t ; g_{2}, g_{3}\right), \quad \bar{p}_{0}(t)=\dot{\bar{q}}_{0}(t), \quad q_{j}=p_{j}=0, \quad j=1, \ldots, N_{f},
$$

where $\wp\left(t ; g_{2}, g_{3}\right)$ is the Weierstrass elliptic function satisfying

$$
\Gamma: \dot{v}^{2}=4 v^{3}-g_{2} v-g_{3}
$$

with $g_{2}=\frac{16}{3} \omega_{0}^{2}-4 h, g_{3}=4 C_{0}^{2}-\frac{8}{3} \omega_{0} h+\frac{64}{27} \omega_{0}^{3}$ and $h$ is level of the Hamiltonian (1.8), chosen so that $\Delta=g_{2}^{3}-27 g_{3}^{2} \neq 0$.

Proof. We put $q_{j}=p_{j}=0, j=1, \ldots, N_{f}$ (recall $C_{j}=0$ ) in (1.8) to obtain

$$
H=\frac{p_{0}^{2}}{2}+\omega_{0} q_{0}^{2}-\frac{q_{0}^{4}}{2}+\frac{C_{0}^{2}}{2 q_{0}^{2}}=\frac{h}{2}
$$

We rewrite this expression in the form

$$
\dot{q}_{0}^{2}=-2 \omega_{0} q_{0}^{2}+q_{0}^{4}-\frac{C_{0}^{2}}{q_{0}^{2}}+h .
$$

Then denoting $u=q_{0}^{2}$ and also $u=v+\frac{2}{3} \omega_{0}$ we obtain the general solution of (3.14)

$$
\bar{q}_{0}^{2}(t)=\frac{2}{3} \omega_{0}+\wp\left(t-t_{0} ; g_{2}, g_{3}\right), \quad \bar{p}_{0}(t)=\dot{\bar{q}}_{0}(t)
$$

We set $t_{0}=0$ to get the desired result.

Next we write the variational equations (VE) along the particular solution (3.11). Denote $\xi_{0}=d q_{0}, \eta_{0}=d p_{0}, \xi_{j}=d q_{j}, \eta_{j}=d p_{j}$. Then the (VE) can be written as

$$
\begin{array}{ll}
\dot{\xi}_{0}=\eta_{0}, & \dot{\eta}_{0}=\left(-2 \omega_{0}+6 \bar{q}_{0}^{2}(t)-\frac{3 C_{0}^{2}}{\bar{q}_{0}^{4}(t)}\right) \xi_{0}, \\
\dot{\xi}_{j}=\eta_{j}, & \dot{\eta}_{j}=\left(-2 \omega_{j}+2 g_{\mathrm{BF}} \bar{q}_{0}^{2}(t)\right) \xi_{j}, \quad j=1, \ldots, N_{f} .
\end{array}
$$

The equation (3.16) forms the tangent part of (VE) and the equations (3.17) form the normal part of (VE), actually (NVE). It is seen from (3.17) that the (NVE) 
splits into a system of $N_{f}$ independent equations $\left(\mathrm{NVE}_{j}\right), j=1, \ldots, N_{f}$. Hence, the (NVE) is integrable if, and only if, each of the $\left(\mathrm{NVE}_{j}\right)$ is integrable. In other words, the identity component of the Galois group of the (NVE) is solvable (commutative) if, and only if, each of identity components of the Galois groups of the $\left(\mathrm{NVE}_{j}\right)$ is solvable (commutative). Therefore, it is enough to study one of them. Let us write the $\left(\mathrm{NVE}_{j}\right)$ for certain particular $j$ as a second order equation

$$
\ddot{\xi}_{j}+\left(2 \omega_{j}-2 g_{\mathrm{BF}} \bar{q}_{0}^{2}(t)\right) \xi_{j}=0 .
$$

Taking into account the particular solution (3.11) the Eq. (3.18) is a Lamé equation

$$
\ddot{\xi}_{j}+\left(2 \omega_{j}-\frac{4}{3} g_{\mathrm{BF}} \omega_{0}-2 g_{\mathrm{BF} \wp}(t)\right) \xi_{j}=0 .
$$

Further, we study the tangential part of the (VE) - Eq. (3.16). The theory gives that its Galois group is solvable. In fact, we have

Proposition 3. The Galois group of (3.16) is abelian.

Proof. It is well known that the system (3.16) has a particular solution $\left(\xi_{0,1}, \dot{\xi}_{0,1}\right)=$ $\left(\overline{p_{0}}(t), \dot{\overline{p_{0}}}(t)\right)$. The other solution is obtained via D'Alembert's formula

$$
\xi_{0,2}=\xi_{0,1} \int_{0}^{t} \frac{d \tau}{\left(\xi_{0,1}\right)^{2}}
$$

Denote the coefficient field of (3.16) by $K=\mathbb{C}\left(\wp(t), \wp^{\prime}(t)\right)$. This field is isomorphic to the field of meromorphic functions $\mathcal{M}(\Gamma)$ on $\Gamma$.

It can be seen from the obtained solutions that one part of them lie in a quadratic extension of the field $K$ and the another part is obtained with single quadrature of the elements of this extension. Therefore the Galois group of (3.16) acts in the following way: $\sigma \in \operatorname{Gal}(L / K), \sigma\left(\xi_{0,1}\right)=\xi_{0,1}$ and $\sigma\left(\xi_{0,2}\right)=\xi_{0,2}+\nu_{0} \xi_{0,1}, \alpha_{0} \in \mathbb{C}$. Let $\Xi(t)$ is the fundamental matrix of (3.16)

$$
\Xi=\left(\begin{array}{cc}
\xi_{0,2} & \xi_{0,1} \\
\dot{\xi}_{0,2} & \dot{\xi}_{0,1}
\end{array}\right)
$$

Then $\sigma \in \operatorname{Gal}(L / K)$ can be represented by the matrix $R_{\nu_{0}}, \sigma \Xi(t)=\Xi(t) R_{\nu_{0}}$, where $R_{\nu_{0}}=\left(\begin{array}{cc}1 & 0 \\ \nu_{0} & 1\end{array}\right)$. It is clear that the group $\left\{\left(\begin{array}{cc}1 & 0 \\ \nu_{0} & 1\end{array}\right)\right\}$ is abelian. 
Now we pass to the analysis of the normal part of the variational equations given by (3.19). Usually the parameter $A$ in the Lamé equation $\ddot{\xi}-\left(A_{\wp}(t)+B\right) \xi=0$ is replaced by a parameter $n$, where $A=n(n+1)$, and thus for (3.19) we have

$$
g_{\mathrm{BF}}=\frac{n(n+1)}{2}, \quad n \in \mathbb{R}
$$

and (recall that the parameters are real)

$$
\ddot{\xi}_{j}-\left[n(n+1) \wp(t)+B_{j}\right] \xi_{j}=0,
$$

where $B_{j}=\frac{2}{3} \omega_{0} n(n+1)-2 \omega_{j}$. The cases for which the Lamé equation (3.21) is solvable are well known:

(i) The Lamé and Hermite solutions. In this case $n \in \mathbb{Z}$ and $g_{2}, g_{3}, B_{j}$ are arbitrary parameters;

(ii) The Brioschi-Halphen-Crowford solutions. Here $m:=n+1 / 2 \in \mathbb{N}$ and the parameters $g_{2}, g_{3}, B_{j}$ must satisfy an algebraic equation.

(iii) The Baldassarri solutions. Now $n+1 / 2 \in \frac{1}{3} \mathbb{Z} \cup \frac{1}{4} \mathbb{Z} \cup \frac{1}{5} \mathbb{Z} \backslash \mathbb{Z}$ with additional algebraic relations between the other parameters.

Note that in the case (i) the identity component of the Galois group $G^{0}$ is of the form $\left(\begin{array}{cc}1 & 0 \\ \nu_{j} & 1\end{array}\right)$ and in the cases (ii) and (iii) $G^{0}=i d$ ( $G$ is finite). And these are the all cases when the Lamé equation is integrable.

Therefore, together with the result of Proposition 2 we have that the identity component of Galois group of the (VE) is represented by the block-diagonal matrices of the kind

$$
\left(\begin{array}{ccccccc}
1 & 0 & 0 & 0 & & 0 & 0 \\
\nu_{0} & 1 & 0 & 0 & \cdots & 0 & 0 \\
\cdots & \cdots & \cdots & \cdots & \\
0 & 0 & 1 & 0 & & 0 & 0 \\
0 & 0 & \nu_{j} & 1 & \cdots & 0 & 0 \\
\cdots & \cdots & & \cdots & \cdots & \cdots \\
0 & 0 & 0 & 0 & \cdots & 1 & 0 \\
0 & 0 & 0 & 0 & \cdots & \nu_{N_{f}} & 1
\end{array}\right)
$$

and it is clearly commutative.

The integrability of Hamiltonian systems with two degrees of freedom which (NVE) are Lamé equations is studied in [11,12]. We summarize the facts and the result (Theorem 5), that gives necessary conditions for integrability in an Appendix. Since in our case the (NVE) splits into a system of $N_{f}$ equations, the result for two degrees of freedom can be applied. 
The potential $\varphi\left(q_{0}\right)$ is obtained from (3.14). Denote $\alpha(t, h):=n(n+1) \wp(t)+B_{j}$. We calculate the coefficients of the polynomial $P(\alpha, h)$ (compare with (A.4) and $(\mathrm{A} .5))$

$$
P(\alpha, h)=\left(a_{1}+h a_{2}\right) \alpha^{3}+\left(b_{1}+h b_{2}\right) \alpha^{2}+\left(c_{1}+h c_{2}\right) \alpha+\left(d_{1}+h d_{2}\right) .
$$

In our case these coefficients are:

$$
\begin{gathered}
a_{1}=\frac{4}{n(n+1)}, \quad a_{2}=0, \quad b_{1}=-\frac{12 B_{j}}{n(n+1)}, \quad b_{2}=0 \\
c_{1}=\frac{12 B_{j}^{2}}{n(n+1)}-\frac{16}{3} \omega_{0}^{2} n(n+1), \quad c_{2}=4 n(n+1), \\
d_{1}=\frac{16}{3} b_{j} \omega_{0}^{2} n(n+1)-\frac{4}{n(n+1)} B_{j}^{3}-n^{2}(n+1)^{2}\left(4 C_{0}^{2}+\frac{64}{27} \omega_{0}^{3}\right), \quad d_{2}=8 n(n+1) \omega_{j} .
\end{gathered}
$$

Now we are ready to apply Theorem 5 (see the Appendix). Suppose that $g_{\mathrm{BF}}=$ $n(n+1) / 2 \neq 0$.

The condition 3 is not fulfilled: $c_{2} \neq 0$ and $c_{2} b_{1}-3 a_{1} d_{2}=-32 \omega_{0} n(n+1)$, which is nonzero by the assumption that $\omega_{0}, \omega_{j}$ are positive numbers, made in the very beginning. In particular, there are no Baldassarri solutions.

We proceed with the cases of the condition 2. In the case $2.1, m=1, b_{1}=0$ is equivalent to

$$
B_{j}=0, \quad \text { or equivalently, } \omega_{j}=\omega_{0} / 4, \quad j=1, \ldots, N_{f} .
$$

If for some $j B_{j} \neq 0$ then the system is not integrable for this $m$. We will consider the case when all $B_{j}=0$ in what follows.

The case $2.2 m=2$ does not occur here since $c_{2} \neq 0$.

In the case $2.3, m=3$ the necessary conditions

$$
16 a_{1} d_{2}+11 b_{1} c_{2}=0, \quad 1024 a_{1}^{2} d_{1}+704 a_{1} b_{1} c_{1}+45 b_{1}^{3}=0
$$

yield correspondingly

$$
B_{j}=\frac{32}{33} \omega_{j}\left(55 \omega_{0}=28 \omega_{j}\right), \quad 7^{3} C_{0}^{2}=72 \omega_{0}^{3} .
$$

If any of the above conditions is violated, then the system is non-integrable for this $m$. We will consider the case when relations (3.24) are valid for all $j$ in what follows.

The case $2 . \mathrm{m}, m>3$ does not occur here since $c_{2} \neq 0$ and $d_{2} \neq 0$.

Finally, if $n \notin \mathbb{Z}$ the condition 1 is not fulfilled. 
In order to resolve the case 2.1 with (3.23) and the case 2.3 with (3.24) of the condition 2 in the Theorem 5 we need to study the Galois groups of higher variational equations and to apply Theorem 3. To compute higher variations we put

$$
\begin{aligned}
q_{0} & =\bar{q}_{0}+\varepsilon \xi_{0}^{(1)}+\varepsilon^{2} \xi_{0}^{(2)}+\varepsilon^{3} \xi_{0}^{(3)}+\ldots, \\
p_{0} & =\bar{p}_{0}+\varepsilon \eta_{0}^{(1)}+\varepsilon^{2} \eta_{0}^{(2)}+\varepsilon^{3} \eta_{0}^{(3)}+\ldots, \\
q_{j} & =0+\varepsilon \xi_{j}^{(1)}+\varepsilon^{2} \xi_{j}^{(2)}+\varepsilon^{3} \xi_{j}^{(3)}+\ldots, \\
p_{j} & =0+\varepsilon \eta_{j}^{(1)}+\varepsilon^{2} \eta_{j}^{(2)}+\varepsilon^{3} \eta_{j}^{(3)}+\ldots, \quad j=1, \ldots, N_{f} .
\end{aligned}
$$

and substitute these expressions into the original Hamiltonian system. Comparing the terms with the same order in $\varepsilon$, we get consecutively the variational equations up to order 3 .

The first variational equation is

$$
\begin{array}{ll}
\dot{\xi}_{0}^{(1)}=\eta_{0}^{(1)}, & \dot{\eta}_{0}^{(1)}=\left(-2 \omega_{0}+6 \bar{q}_{0}^{2}-\frac{3 C_{0}^{2}}{\bar{q}_{0}^{4}}\right) \xi_{0}^{(1)}, \\
\dot{\xi}_{j}^{(1)}=\eta_{j}^{(1)}, & \dot{\eta}_{j}^{(1)}=\left(-2 \omega_{j}+2 g_{\mathrm{BF}} \bar{q}_{0}^{2}\right) \xi_{j}^{(1)}, \quad j=1, \ldots, N_{f},
\end{array}
$$

but of course we know it (see $(3.16,(3.17))$. For the second variational equation we have

$$
\begin{array}{ll}
\dot{\xi}_{0}^{(2)}=\eta_{0}^{(2)}, & \dot{\eta}_{0}^{(2)}=\left(-2 \omega_{0}+6 \bar{q}_{0}^{2}-\frac{3 C_{0}^{2}}{\bar{q}_{0}^{4}}\right) \xi_{0}^{(2)}+K_{0}^{(2)}, \\
\dot{\xi}_{j}^{(2)}=\eta_{j}^{(2)}, & \dot{\eta}_{j}^{(2)}=\left(-2 \omega_{j}+2 g_{\mathrm{BF}} \bar{q}_{0}^{2}\right) \xi_{j}^{(2)}+K_{j}^{(2)}, \quad j=1, \ldots, N_{f} .
\end{array}
$$

The third variational equation is

$$
\begin{array}{ll}
\dot{\xi}_{0}^{(3)}=\eta_{0}^{(3)}, & \dot{\eta}_{0}^{(3)}=\left(-2 \omega_{0}+6 \bar{q}_{0}^{2}-\frac{3 C_{0}^{2}}{\bar{q}_{0}^{4}}\right) \xi_{0}^{(3)}+K_{0}^{(3)}, \\
\dot{\xi}_{j}^{(3)}=\eta_{j}^{(3)}, & \dot{\eta}_{j}^{(3)}=\left(-2 \omega_{j}+2 g_{\mathrm{BF}} \bar{q}_{0}^{2}\right) \xi_{j}^{(3)}+K_{j}^{(3)}, \quad j=1, \ldots, N_{f} .
\end{array}
$$


Here

$$
\begin{aligned}
K_{0}^{(2)} & =2 g_{\mathrm{BF}} \bar{q}_{0} \sum\left(\xi_{j}^{(1)}\right)^{2}+6 \bar{q}_{0}\left(\xi_{0}^{(1)}\right)^{2}+6 C_{0}^{2} \frac{\left(\xi_{0}^{(1)}\right)^{2}}{\bar{q}_{0}^{5}} \\
K_{j}^{(2)} & =4 g_{\mathrm{BF}} \bar{q}_{0} \xi_{0}^{(1)} \xi_{j}^{(1)}, \quad j=1, \ldots, N_{f}, \\
K_{0}^{(3)} & =2 g_{\mathrm{BF}}\left[2 \bar{q}_{0} \sum \xi_{j}^{(1)} \xi_{j}^{(2)}+\xi_{0}^{(1)} \sum\left(\xi_{j}^{(1)}\right)^{2}\right]+2\left(\xi_{0}^{(1)}\right)^{3}+12 \bar{q}_{0} \xi_{0}^{(1)} \xi_{0}^{(2)} \\
& -\frac{C_{0}^{2}}{\bar{q}_{0}^{6}}\left[10\left(\xi_{0}^{(1)}\right)^{3}-12 \bar{q}_{0} \xi_{0}^{(1)} \xi_{0}^{(2)}\right], \\
K_{j}^{(3)} & =2 g_{\mathrm{BF}}\left[\left(\xi_{0}^{(1)}\right)^{2} \xi_{j}^{(1)}+2 \bar{q}_{0}\left(\xi_{0}^{(1)} \xi_{j}^{(2)}+\xi_{0}^{(2)} \xi_{j}^{(1)}\right)\right], \quad j=1, \ldots, N_{f} .
\end{aligned}
$$

Then, in our notation from Section 2, we have

$$
\begin{aligned}
& f_{2}=\left[0, K_{0}^{(2)}, 0, K_{1}^{(2)}, \ldots, 0, K_{N_{f}}^{(2)}\right]^{T} \\
& f_{3}=\left[0, K_{0}^{(3)}, 0, K_{1}^{(3)}, \ldots, 0, K_{N_{f}}^{(3)}\right]^{T}
\end{aligned}
$$

First, we have to solve $\left(\mathrm{VE}_{1}\right)$. Let $\xi_{0,1}^{(1)}, \xi_{0,2}^{(1)}$ be two linearly independent solutions of (3.26) with Wronskian equal to unity, i.e., $\xi_{0,1}^{(1)} \dot{\xi}_{0,2}^{(1)}-\dot{\xi}_{0,1}^{(1)} \xi_{0,2}^{(1)}=1$. Similarly, $\xi_{j, 1}^{(1)}, \xi_{j, 2}^{(1)}$ are linearly independent solutions of (3.27) with Wronskian equal to unity. Then the fundamental matrix $X(t)$ of $(3.26),(3.27)$ and its inverse have the block-diagonal form

$$
X(t)=\left(\begin{array}{ccccccc}
\xi_{0,1}^{(1)} & \xi_{0,2}^{(1)} & 0 & 0 & & 0 & 0 \\
\dot{\xi}_{0,1}^{(1)} & \dot{\xi}_{0,2}^{(1)} & 0 & 0 & \cdots & 0 & 0 \\
\ldots & \ldots & \ldots & \ldots \\
0 & 0 & \xi_{j, 1}^{(1)} & \xi_{j, 2}^{(1)} & & 0 & 0 \\
0 & 0 & \dot{\xi}_{j, 1}^{(1)} & \dot{\xi}_{j, 2}^{(1)} & \ldots & 0 & 0 \\
\ldots & & \ldots & \ldots & & \ldots \\
0 & 0 & 0 & 0 & & \xi_{N_{f}, 1}^{(1)} & \xi_{N_{f}, 2}^{(1)} \\
0 & 0 & 0 & 0 & \cdots & \dot{\xi}_{N_{f}, 1}^{(1)} & \dot{\xi}_{N_{f}, 2}^{(1)}
\end{array}\right),
$$




$$
X^{-1}(t)=\left(\begin{array}{ccccccc}
\dot{\xi}_{0,2}^{(1)} & -\xi_{0,2}^{(1)} & 0 & 0 & & 0 & 0 \\
-\dot{\xi}_{0,1}^{(1)} & \xi_{0,1}^{(1)} & 0 & 0 & \cdots & 0 & 0 \\
\ldots & \ldots & \ldots & \ldots \\
0 & 0 & \dot{\xi}_{j, 2}^{(1)} & -\xi_{j, 2}^{(1)} & \ldots & 0 & 0 \\
0 & 0 & -\dot{\xi}_{j, 1}^{(1)} & \xi_{j, 1}^{(1)} & \cdots & 0 & 0 \\
\cdots & \ldots & \ldots & \ldots & \ldots \\
0 & 0 & 0 & 0 & & \dot{\xi}_{N_{f}, 2}^{(1)} & -\xi_{N_{f}, 2}^{(1)} \\
0 & 0 & 0 & 0 & \cdots & -\dot{\xi}_{N_{f}, 1}^{(1)} & \xi_{N_{f}, 1}^{(1)}
\end{array}\right)
$$

The first variational equations $\left(\mathrm{VE}_{1}\right)(3.26)$, (3.27) have a singular point at $t=0$ (the pole of $\wp(t)$ ). We calculate the expansion of the solutions of variational equations around the point $t=0$. Note that

$$
\bar{q}_{0}(t)=\frac{1}{t}+\frac{\omega_{0}}{3} t+\left(\frac{g_{2}}{40}-\frac{\omega_{0}^{2}}{18}\right) t^{3}+\ldots
$$

Here and further dots denote the higher order terms with respect to $t$. In a neighborhood of $t=0$ we have the following expansions for the solutions of the tangential part of $\left(\mathrm{VE}_{1}\right)$ Eq. (3.26)

$$
\xi_{0,1}^{(1)}=\frac{1}{t^{2}}-\frac{\omega_{0}}{3}-\left(\frac{3 g_{2}}{40}-\frac{\omega_{0}^{2}}{6}\right) t^{2}+\ldots, \quad \xi_{0,2}^{(1)}=\frac{t^{3}}{5}+\frac{\omega_{0}}{35} t^{5}+\ldots
$$

After this preparation, we are ready to proceed with the cases unresolved from the analysis of the first variational equations $\left(\mathrm{VE}_{1}\right)$.

We continue the study of the case 2.1 with (3.23). Here $n=\frac{1}{2}$ and $g_{\mathrm{BF}}=\frac{3}{8}$. One can find the following expansions of the solutions of (3.27) in this case

$$
\xi_{j, 1}^{(1)}=t^{-1 / 2}\left(1+\frac{3}{640} g_{2} t^{4}+\ldots\right), \quad \xi_{j, 2}^{(1)}=t^{3 / 2}\left(\frac{1}{2}+\frac{1}{2.640} g_{2} t^{4}+\ldots\right) .
$$

There are no logarithms in the expansions around $t=0$ of the local solutions of the second variational equation $\left(\mathrm{VE}_{2}\right)$ due to $(3.23)$. We take

$$
\xi_{0}^{(1)}=\xi_{0,1}^{(1)}, \quad \xi_{j}^{(1)}=\xi_{j, 2}^{(1)}
$$

With the above choice, we find that

$$
\xi_{0}^{(2)}=\xi_{0,2}^{(2)}=\frac{1}{t^{3}}+O(t), \quad \xi_{j}^{(2)}=\xi_{j, 1}^{(2)}=\frac{1}{\sqrt{t}}-\frac{3}{4} \sqrt{t}+O\left(t^{3 / 2}\right) .
$$


We will show that a logarithmic term appears in a local solution of $\left(\mathrm{VE}_{3}\right)$. For this purpose, it is enough to show that at least one component of $X^{-1} f_{3}$ has a nonzero residue at $t=0$, see formulae $(2.7),(2.8)$. We calculate $j$-th component $j=1, \ldots, N_{f}$ of $X^{-1} f_{3}$, which looks like

$$
\left(-\xi_{j, 2}^{(1)} K_{j}^{(3)}, \xi_{j, 1}^{(1)} K_{j}^{(3)}\right)^{T} .
$$

Then the first term in (3.38) has the following expansion around $t=0$

$$
\mu_{3}=-\xi_{j, 2}^{(1)} K_{j}^{(3)}=-\frac{3}{8}\left[\frac{2}{t^{2}}-\frac{2 \omega_{0}}{3 t}+\ldots\right]
$$

that is, $\mu_{3}$ has a pole at $t=0$ with non-zero residue $\frac{\omega_{0}}{4}$. Therefore, the identity component of the Galois group of $\left(\mathrm{VE}_{3}\right)$ is not abelian and hence, in this case, the Hamiltonian system (1.8) is not integrable due to Theorem 3.

Next we consider the case 2.3 with (3.24). Here $n=\frac{5}{2}$ and $g_{\mathrm{BF}}=\frac{35}{8}$. One can find the following expansions of the solutions of (3.27) in this case

$$
\xi_{j, 1}^{(1)}=t^{-5 / 2}\left(\frac{1}{6}+O\left(t^{2}\right)\right), \quad \xi_{j, 2}^{(1)}=t^{7 / 2}\left(1+O\left(t^{2}\right)\right) .
$$

There are no logarithms in the expansions around $t=0$ of the local solutions of the second variational equation $\left(\mathrm{VE}_{2}\right)$ due to $(3.24)$.

We take

$$
\xi_{0}^{(1)}=\xi_{0,1}^{(1)}, \quad \xi_{j}^{(1)}=\xi_{j, 1}^{(1)} .
$$

With the above choice, we find that

$$
\begin{gathered}
\xi_{0}^{(2)}=\xi_{0,2}^{(2)}=\frac{5 N_{f}}{144 t^{4}}+\frac{1}{t^{3}}-\frac{N_{f} \omega_{0}}{224 t^{2}}-\frac{\omega_{0}}{5 t}+O\left(t^{0}\right), \\
\xi_{j}^{(2)}=\xi_{j, 2}^{(2)}=t^{7 / 2}\left(1+O\left(t^{2}\right)+t^{-7 / 2}\left(\frac{5}{12}-\frac{\omega_{j}}{99} t^{2}+\ldots\right) .\right.
\end{gathered}
$$

Again the first term in (3.38) has the expansion around $t=0$

$$
\mu_{3}=-\xi_{j, 2}^{(1)} K_{j}^{(3)}=-\frac{175 N_{f}}{1728 t^{4}}-\frac{35}{3 t^{3}}-\frac{5 N_{f} \omega_{0}}{576 t^{2}}+\frac{7 \omega_{0}}{12 t}+O\left(t^{0}\right)
$$

that is, $\mu_{3}$ has a non-zero residue $\frac{7}{12} \omega_{0}$ at $t=0$. Therefore, the identity component of the Galois group of $\left(\mathrm{VE}_{3}\right)$ is not abelian and hence, in this case, the Hamiltonian system (1.8) is not integrable due to Theorem 3 .

This finishes the proof of this part of Theorem 1 . 


\subsection{The case $C_{0} \neq 0, C_{1} \neq 0$.}

Here we consider the Hamiltonian (1.8) only for two degrees of freedom (see comments in the next section)

$$
H=\frac{p_{0}^{2}}{2}+\omega_{0} q_{0}^{2}-\frac{q_{0}^{4}}{2}+\frac{C_{0}^{2}}{2 q_{0}^{2}}+\frac{p_{1}^{2}}{2}+\omega_{1} q_{1}^{2}+\frac{C_{1}^{2}}{2 q_{1}^{2}}-g_{\mathrm{BF}} q_{0}^{2} q_{1}^{2} .
$$

Denote $\varepsilon:=g_{\mathrm{BF}}$ and assume that $\varepsilon$ is small enough. We can rewrite (3.39) as

$$
H=H_{0}+\varepsilon H_{1}
$$

where

$$
H_{0}=\frac{p_{0}^{2}}{2}+\omega_{0} q_{0}^{2}-\frac{q_{0}^{4}}{2}+\frac{C_{0}^{2}}{2 q_{0}^{2}}+\frac{p_{1}^{2}}{2}+\omega_{1} q_{1}^{2}+\frac{C_{1}^{2}}{2 q_{1}^{2}}, \quad H_{1}=-q_{0}^{2} q_{1}^{2}
$$

The unperturbed system $(\varepsilon=0)$ is separable.

$$
\begin{aligned}
& \dot{q}_{0}=p_{0}, \quad \dot{p}_{0}=-2 \omega_{0} q_{0}+2 q_{0}^{3}+\frac{C_{0}^{2}}{q_{0}^{3}} \\
& \dot{q}_{1}=p_{1}, \quad \dot{p}_{1}=-2 \omega_{1} q_{1}+\frac{C_{1}^{2}}{q_{1}^{3}}
\end{aligned}
$$

From the proof of Proposition 2 the general solution of (3.42) is found in (3.15). From the proof of Proposition 1 the general solution of (3.43) is

$$
q_{1}^{2}=\frac{h_{1}}{2 \omega_{1}}+\sqrt{\frac{C_{1}^{2}}{2 \omega_{1}}-\frac{h_{1}^{2}}{4 \omega_{1}^{2}}} \sinh 2 i \sqrt{2 \omega_{1}}\left(t-t_{0}\right), \quad p_{1}=\dot{q}_{1} .
$$

First, we put the Hamiltonian $H$ in the context of the theory recalled in Section 2. It is assumed that at this point the variables are real. We introduce action-angle variables $(I, \varphi)$, so that $H_{0}=H_{0}\left(q_{0}, p_{0}, I\right)$. To do so, we need to find a generating function $S\left(I, q_{1}\right)$ :

$$
\left(p_{1}, q_{1}\right) \stackrel{S\left(I, q_{1}\right)}{\longrightarrow}(I, \varphi), \quad p_{1}=\frac{\partial S}{\partial q_{1}}, \quad \varphi=\frac{\partial S}{\partial I}
$$

such that

$$
\frac{p_{1}^{2}}{2}+\omega_{1} q_{1}^{2}+\frac{C_{1}^{2}}{2 q_{1}^{2}}=h_{1} \rightarrow h_{1}(I):=I
$$


Note that the real ovals for the curve $\left(p_{1}, q_{1}\right)$ in $(3.45)$ exist for $h_{1}>\frac{C_{1}^{2} \sqrt{2 \omega_{1}}}{\sqrt{C_{1}^{2}}}$. Then the formula (3.44) becomes

$$
q_{1}^{2}=\frac{h_{1}}{2 \omega_{1}}-\sqrt{\frac{h_{1}^{2}}{4 \omega_{1}^{2}}-\frac{C_{1}^{2}}{2 \omega_{1}}} \sin 2 \sqrt{2 \omega_{1}}\left(t-t_{0}\right) .
$$

The generating function $S$ can be found explicitly, but we do not need it, we just set

$$
I:=\frac{p_{1}^{2}}{2}+\omega_{1} q_{1}^{2}+\frac{C_{1}^{2}}{2 q_{1}^{2}}, \quad \varphi:=\int \frac{d q_{1}}{p_{1}} .
$$

Note that $d I \wedge d \varphi=d p_{1} \wedge d q_{1}, \varphi$ is multivalued, but $\dot{\varphi}=1$, that is, $t$ and $\varphi$ are interchangeable.

Next, we fix $I$ to an arbitrary constant greater than $\frac{C_{1}^{2} \sqrt{2 \omega_{1}}}{\sqrt{C_{1}^{2}}}$ and again consider $t, q_{0}(t), p_{0}(t)$ as complex variables. Our system becomes an one-and-a-half degrees of freedom system with a Hamiltonian $H=H_{0}+\varepsilon H_{1}$, where

$H_{0}=\frac{p_{0}^{2}}{2}+\omega_{0} q_{0}^{2}-\frac{q_{0}^{4}}{2}+\frac{C_{0}^{2}}{2 q_{0}^{2}}+I, \quad H_{1}=-q_{0}^{2}\left(\frac{I}{2 \omega_{1}}-\sqrt{\frac{I^{2}}{4 \omega_{1}^{2}}-\frac{C_{1}^{2}}{2 \omega_{1}}} \sin 2 \sqrt{2 \omega_{1}}\left(t-t_{0}\right)\right)$.

We need to find a separatrix in the dynamics of $\left(q_{0}, p_{0}\right)$. Denote $\tilde{h}=h-I$ and $\tilde{g}_{2}=\frac{16}{3} \omega_{0}^{2}-4 \tilde{h}, \tilde{g}_{3}=4 C_{0}^{2}-\frac{8}{3} \omega_{0} \tilde{h}+\frac{64}{27} \omega_{0}^{3}$ (compare with the corresponding formulas in the Proposition 2). Let $h^{*}$ be the biggest real root of

$$
\Delta(\tilde{h})=\tilde{g}_{2}^{3}-27 \tilde{g}_{3}^{2}=-64\left(\tilde{h}^{3}-\omega_{0}^{2} \tilde{h}^{2}-9 C_{0}^{2} \omega_{0} \tilde{h}+8 C_{0}^{2} \omega_{0}^{3}+\frac{27}{4} C_{0}^{4}\right)=0 .
$$

Assume that $4 \omega_{0}^{2}-3 h^{*}>0$. Further, we denote

$$
a:=\frac{\sqrt{4 \omega_{0}^{2}-3 h^{*}}}{3}>0
$$

Then the unperturbed system (3.48) has a separatrix

$$
\Gamma_{0}: q_{0}^{2}(t)=\frac{2}{3} \omega_{0}+a+\frac{3 a}{\sinh ^{2}(\sqrt{3 a} t)}, \quad p_{0}(t)=\dot{q}_{0}(t)
$$

The perturbed variational equation (PVE) of (3.48) along $\Gamma_{t_{0}}$ is given by (see $\left.[7,15]\right)$

$$
\frac{d}{d t}\left(\begin{array}{l}
\xi \\
\eta \\
\nu
\end{array}\right)=\left(\begin{array}{ccc}
H_{0, q_{0} p_{0}} & H_{0, p_{0} p_{0}} & H_{1, p_{0}} \\
-H_{0, q_{0} q_{0}} & -H_{0, q_{0} p_{0}} & -H_{1, q_{0}} \\
0 & 0 & 0
\end{array}\right)\left(\begin{array}{l}
\xi \\
\eta \\
\nu
\end{array}\right)
$$


where all coefficients are restricted to $\Gamma_{t_{0}}$. In order to study the Galois group of (PVE) we fix the coefficient field $K$ in (3.51). From the expressions for the separatrix (3.50) and the perturbation $H_{1}(3.48)$

$$
K:=\mathbb{C}\left(e^{\sqrt{3 a} t}, e^{2 \sqrt{2 \omega_{1}} i t}\right) .
$$

Then, to obtain the fundamental matrix of (3.51) a quadrature is needed, namely $\delta=\delta(t)=\left.\int \frac{H_{0, p_{0} p_{0}}}{H_{0, p_{0}}^{2}}\right|_{\Gamma_{0}} d t$ (see [15] for details). In our case $\delta=\int \frac{d t}{p_{0}^{2}(t)}$ equals

$$
\begin{array}{r}
\delta=\frac{1}{(3 a)^{3}}\left(\frac{2 \omega_{0}+3 a}{12 \sqrt{3 a}} \sinh (\sqrt{3 a} t) \cosh ^{3}(\sqrt{3 a} t)+\frac{10 \omega_{0}+27 a}{8 \sqrt{3 a}} \sinh (\sqrt{3 a} t) \cosh (\sqrt{3 a} t)\right. \\
\left.+\frac{2 \omega_{0}+12 a}{3 \sqrt{3 a}} \tanh (\sqrt{3 a} t)+\frac{26 \omega_{0}+99 a}{8} t\right) .
\end{array}
$$

It is clear that $\delta=\delta(t)$ is uniform and $\delta \notin K$. Then, the Picard-Vessiot extension of $(3.51)$ is $L_{1}=K(\delta)=\mathbb{C}\left(e^{\sqrt{3 a} t}, e^{2 \sqrt{2 \omega_{1}} i t}, t\right)$. It remains to find $d\left(t_{0}\right)$. Let $\gamma$ be a loop around the pole $t=0$. Then simple calculations give that the Poincaré-ArnoldMelnikov integral is

$$
d\left(t_{0}\right)=\int_{\gamma}\left\{H_{0}, H_{1}\right\}\left(q_{0}(t), p_{0}(t), t-t_{0}\right) d t=12 \pi i a \sqrt{2 \omega_{1}} \sqrt{\frac{I^{2}}{4 \omega_{1}^{2}}-\frac{C_{1}^{2}}{2 \omega_{1}}} \sin 2 \sqrt{2 \omega_{1}} t_{0} .
$$

It is seen that $d\left(t_{0}\right)$ has simple zeroes and by Theorem 4 , the perturbed separatrix self-intersects transversally. Also since $d\left(t_{0}\right)$ is not identically zero, the Galois group of the perturbed variational equation is not abelian [15]. Hence, when $\varepsilon=g_{\mathrm{BF}} \neq 0$ sufficiently small, there is no additional meromorhic first integral. This finishes the proof of this part and therefore, the proof of the Theorem 1.

\section{Concluding Remarks}

In this paper we use variational equations to obtain a necessary and sufficient condition for integrability of a system which describes the stationary solutions in the time dependent mean field equations of Bose-Fermi mixture. Here we make some remarks.

We start with some restrictions to our methods. In subsection 3.1 we don't know how to study the Galois group of a second order linear equation with quasi-periodic coefficient, that is why we assume that all $\omega_{j}$ are equal. It is an open problem to 
develop a Picard-Vessiot Theory for the coefficient field $K=\mathbb{C}\left(e^{\alpha_{1} x}, \ldots, e^{\alpha_{m} x}\right)$ with $\alpha_{1}, \ldots, \alpha_{m} \in \mathbb{C}$ and to relate this result with the integrability of the corresponding linear equation, see [18], p. 408.

In the second part of Theorem 1 considered in the subsection 3.2, it turns out that the (NVE) splits into a system of second order linear equations of Lamé type. By applying Theorem 5 and by studying the Galois groups of the variational equations up to order three, we prove that the Hamiltonian system (1.8) is non-integrable when $g_{\mathrm{BF}}=n(n+1) / 2, n \notin \mathbb{Z}$. Unfortunately, we do not give a complete answer for the integrability of the system (1.8) in the Lamé and Hermite case when $g_{\mathrm{BF}}=$ $n(n+1) / 2, n \in \mathbb{Z}$. That is why we have formulated the Conjecture in the Introduction. We consider only $n=1$ and $n=2$ by technical reasons. As it is seen from subsection 3.2 and the Appendix, in the Lamé and Hermite case it is not possible to derive additional integrability conditions from the analysis of the $\left(\mathrm{VE}_{1}\right)$. Therefore, in order to study the integrability for arbitrary $n \in \mathbb{Z}$ in $g_{\mathrm{BF}}=n(n+1) / 2$, one needs to know the exact coefficients in expansions of the Lamé solutions of (3.27) and eventually the expansions of the higher (than order three) variations. The formulas are quite involved. However, it is highly unlikely that the system is integrable for some integer $n>2$, which is justified by the result in 3.3 .

\section{The cases $n=1$ and $n=2$ from the Conjecture.}

We take $n=1$, but we keep writing $g_{\mathrm{BF}}$ instead 1 . In the vicinity $t=0$ we have the following expansions for the solutions $\xi_{j, 1}^{(1)}, \xi_{j, 2}^{(1)}$ of $(3.27)(n=1)$

$$
\xi_{j, 1}^{(1)}=\frac{1}{t}+\frac{B_{j}}{2} t+\left(\frac{g_{2}}{40}-\frac{B_{j}^{2}}{8}\right) t^{3}+\ldots, \quad \xi_{j, 2}^{(1)}=\frac{t^{2}}{3}-\frac{a_{j}}{30} t^{4}+\ldots,
$$

where $B_{j}=2 \omega_{j}-4 \omega_{0} / 3$.

There are no logarithms in the expansions around $t=0$ of the local solutions of the second variational equation $\left(\mathrm{VE}_{2}\right)$.

We take

$$
\xi_{0}^{(1)}=\xi_{0,2}^{(1)}, \quad \xi_{j}^{(1)}=\xi_{j, 1}^{(1)}
$$

With this choice we find

$$
\xi_{0,1}^{(2)}=\frac{1}{t^{2}}+\frac{g_{\mathrm{BF}} N_{f}}{2} \frac{1}{t}-\frac{\omega_{0}}{3}+\ldots, \quad \xi_{0,2}^{(2)}=\frac{g_{\mathrm{BF}} N_{f}}{2} \frac{1}{t}+\ldots
$$

and

$$
\xi_{j, 1}^{(2)}=\frac{1}{t}+\frac{B_{j}}{2} t+\ldots, \quad \xi_{j, 2}^{(2)}=\frac{t^{2}}{3}+\ldots
$$


Taking the first term in (3.38), namely

$$
\mu_{3}=-\xi_{j, 2}^{(1)} K_{j}^{(3)}=-\xi_{j, 2}^{(1)} 2 g_{\mathrm{BF}}\left[\left(\xi_{0}^{(1)}\right)^{2} \xi_{j}^{(1)}+2 \bar{q}_{0}\left(\xi_{0}^{(1)} \xi_{j}^{(2)}+\xi_{0}^{(2)} \xi_{j}^{(1)}\right)\right]
$$

with the choice (4.2) and $\xi_{0}^{(2)}=\xi_{0,2}^{(2)}$ and $\xi_{j}^{(2)}=\xi_{j, 1}^{(2)}$ we can see that $\mu_{3}$ has a simple pole at $t=0$ with residue $-2 g_{\mathrm{BF}}^{2} N_{f} / 3$, which is non-zero. Therefore, the identity component of the Galois group of $\left(\mathrm{VE}_{3}\right)$ is not commutative and hence, in this case, the Hamiltonian system (1.8) is not integrable due to Theorem 3.

Similarly, for $n=2$ and $g_{\mathrm{BF}}=3$ we have the following expansions for the solutions $\xi_{j, 1}^{(1)}, \xi_{j, 2}^{(1)}$ of $(3.27)$

$$
\xi_{j, 1}^{(1)}=\frac{1}{t^{2}}-\frac{B_{j}}{6}+O\left(t^{2}\right), \quad \xi_{j, 2}^{(1)}=\frac{t^{3}}{5}+\frac{B_{j} t^{5}}{70}+\ldots,
$$

where $B_{j}=4 \omega_{0}-2 \omega_{j}(n=2)$. Let us study first the expansions of the local solutions of the $\left(\mathrm{VE}_{2}\right)$ around $t=0$. The calculation of $\mu_{2}=\xi_{j, 1}^{(1)} K_{j}^{(2)}$ with $\xi_{0}^{(1)}=\xi_{0,1}^{(1)}, \xi_{j}^{(1)}=\xi_{j, 1}^{(1)}$ gives

$$
\mu_{2}=\frac{12}{t^{7}}-\frac{4 B_{j}}{t^{5}}+\frac{\frac{4}{3} B_{j}^{2}-\frac{12}{5} g_{2}}{t^{3}}+\frac{B_{j}\left(g_{2}-\frac{1}{3} B_{j}^{2}\right)}{t}+O(t) .
$$

Since $g_{2}$ depends on $h$, which is arbitrary provided $\Delta=g_{2}^{3}-27 g_{3}^{2} \neq 0$, the only possibility for the residue of $\mu_{2}$ to be zero is $B_{j}=0$, or equivalently, $\omega_{j}=2 \omega_{0}$. If there exists at least one $\omega_{j}$, such that $B_{j} \neq 0$, then a logarithm appears in the solutions of $\left(\mathrm{VE}_{2}\right)$ around $t=0$.

We proceed with the case when all $B_{j}=0$, or equivalently, $\omega_{j}=2 \omega_{0}, j=$ $1, \ldots, N_{f}$. Choosing

$$
\xi_{0}^{(1)}=\xi_{0,1}^{(1)}=\frac{1}{t^{2}}-\frac{\omega_{0}}{3}+\ldots, \quad \xi_{j}^{(1)}=\xi_{j, 2}^{(1)}=\frac{t^{3}}{5}+\ldots
$$

we find that

$$
\xi_{0,1}^{(2)}=\frac{1}{t^{3}}+\frac{1}{t^{2}}-\frac{\omega_{0}}{5 t}-\frac{\omega_{0}}{3}+\ldots, \quad \xi_{0,2}^{(2)}=\frac{1}{t^{3}}-\frac{\omega_{0}}{5 t}+\ldots
$$

and

$$
\xi_{j, 1}^{(2)}=\frac{1}{t^{2}}+\ldots, \quad \xi_{j, 2}^{(2)}=-\frac{3}{5} t^{2}+\frac{t^{3}}{5}+\ldots
$$

Taking the second term in (3.38) $\mu_{3}=\xi_{j, 1}^{(1)} K_{j}^{(3)}$ with the choice (4.6) and $\xi_{0}^{(2)}=$ $\xi_{0,2}^{(2)}, \xi_{j}^{(2)}=\xi_{j, 2}^{(2)}$ one can see that $\mu_{3}$ has a simple pole with a residue $-\omega_{0} 72 / 25$, which is nonzero since $\omega_{0} \neq 0$ by assumption. 
In either of the cases above, the identity component of the Galois group of $\left(\mathrm{VE}_{2}\right)$ or $\left(\mathrm{VE}_{3}\right)$ is not commutative and the Hamiltonian system (1.8) is not integrable due to Theorem 3.

We notice that the non-integrability result obtained in this part are also valid for the limiting case $C_{0}=0$ and $C_{j}=0, j=1, \ldots, N_{f}$.

In the general case $C_{0} \neq 0$ and $C_{j} \neq 0, j=1, \ldots, N_{f}$ (VE) does not split in nice way as in the previous cases. Because of this reason, we consider the system (1.8) with two degrees of freedom. Even then, studying the Galois group of (NVE) is not so simple due to the number of the parameters. That is why in subsection 3.3 we use a perturbational approach, which is still related to the Differential Galois approach. Furthermore, this approach gives a dynamical meaning to the algebraic obstructions to integrability. Note that, the using Poincaré-Arnold-Melnikov integrals in more degrees of freedom for real Hamiltonian systems needs certain KAM-conditions.

The above results allow us to think that the system (1.8) is not integrable unless $g_{\mathrm{BF}}=0$. Moreover, the formulas (3.4) and (3.15) give the general solution to the separable system $\left(g_{\mathrm{BF}}=0\right)$.

Acknowledgements The authors thank to the anonymous referees for their criticism, remarks and suggestions which significantly improved this paper. O. C. acknowledges funding from Bulgarian NSF Grant DDVU 02/90. 


\section{Appendix A. Necessary conditions for integrability of Hamiltonian sys- tems which have (NVE) of Lamé type}

In this appendix we recall some facts concerning the integrability of Hamiltonian systems with two degrees of freedom, an invariant plane and which (NVE) are of Lamé type. More details can be found in $[11,12]$. In our case the (NVE) splits into a system of $N_{f}$ equations of Lamé type, and therefore, these arguments can be applied.

Classically the Lamé equation is written in the form

$$
\ddot{\xi}-[n(n+1) \wp(t)+B] \xi=0,
$$

where $\wp(t)$ is the Weierstrass function with invariants $g_{2}$ and $g_{3}$, satisfying $\dot{v}^{2}=$ $4 v^{3}-g_{2} v-g_{3}$ with $\Delta=g_{2}^{3}-27 g_{3}^{2} \neq 0$.

The known (mutually exclusive) cases of closed form solutions of (A.1) are:

(i) The Lamé and Hermite solutions. In this case $n \in \mathbb{Z}$ and $g_{2}, g_{3}, B$ are arbitrary parameters;

(ii) The Brioschi-Halphen-Crowford solutions. Here $m:=n+1 / 2 \in \mathbb{N}$ and the parameters $g_{2}, g_{3}, B$ must satisfy an algebraic equation.

(iii) The Baldassarri solutions. Now $n+1 / 2 \in \frac{1}{3} \mathbb{Z} \cup \frac{1}{4} \mathbb{Z} \cup \frac{1}{5} \mathbb{Z} \backslash \mathbb{Z}$ with additional algebraic relations between the other parameters.

Note that in the case (i) the identity component of the Galois group $G^{0}$ is of the form $\left(\begin{array}{ll}1 & 0 \\ \nu & 1\end{array}\right)$ and in the cases (ii) and (iii) $G^{0}=i d$ ( $G$ is finite). And these are the all cases when the Lamé equation is integrable.

Now consider a natural two degrees of freedom Hamiltonian

$$
H=\frac{1}{2}\left(p_{1}^{2}+p_{2}^{2}\right)+V\left(q_{1}, q_{2}\right)
$$

$q_{j}(t) \in \mathbb{C}, p_{j}(t)=\dot{q}_{j}, j=1,2$. We assume that there exists a family of solutions of the form

$$
\Gamma_{h}: q_{2}=p_{2}=0, \quad q_{1}=q_{1}(t, h), \quad p_{1}(t, h)=\dot{q}_{1}(t, h)
$$

and $q_{1}(t, h)$ is a solution of

$$
\frac{1}{2} \dot{q}_{1}^{2}+\varphi\left(q_{1}\right)=h, \quad h \in \mathbb{R}
$$

The (NVE) along $\Gamma_{h}$ is

$$
\ddot{\xi}-\alpha(t, h) \xi=0,
$$

where $\alpha(t, h)=\alpha\left(q_{1}(t, h)\right)$ is such that (A.3) is of type (A.1). 
In $[11,12]$ the type of the potentials $V$ with this property are obtained as well as the necessary conditions for the integrability of the Hamiltonian systems with the Hamiltonian (A.2). In order to formulate the result we need certain additional quantities.

Since $\alpha(t, h)$ depends linearly on $\wp(t)$, then $\dot{\alpha}^{2}$ is a cubic polynomial in $\alpha$, depending also in $h$, namely

$$
\dot{\alpha}^{2}:=P(\alpha, h)=P_{1}(\alpha)+h P_{2}(\alpha) .
$$

The following coefficients are introduced

$$
P(\alpha, h)=\left(a_{1}+h a_{2}\right) \alpha^{3}+\left(b_{1}+h b_{2}\right) \alpha^{2}+\left(c_{1}+h c_{2}\right) \alpha+\left(d_{1}+h d_{2}\right) .
$$

Now we are ready to give the corresponding result. Note that the following Theorem gives necessary conditions only from the analysis of the first variational equation.

Theorem 5. (Theorem 6.2 [12]). Assume that a natural Hamiltonian system has (NVE) of Lamé type, associated to the family of solutions $\Gamma_{h}$, lying on the plane $q_{2}=0$ and parametrized by the energy $h$. Then, a necessary conditions for integrability is that the related polynomials $P_{1}$ and $P_{2}$ satisfy $a_{2}=0$, and one of the following conditions holds:

1. $a_{1}=\frac{4}{n(n+1)}$ for some $n \in \mathbb{N}$;

2. $a_{1}=\frac{16}{4 m^{2}-1}$ for some $m \in \mathbb{N}$. Then, assuming the conjecture above is true, one should have $b_{2}=0$ and we should be in one of the following cases:

2.1) $m=1$ and $b_{1}=0$,

2.2) $m=2$ and $c_{2}=0,16 a_{1} c_{1}+3 b_{1}^{2}=0$,

2.3) $m=3$ and $16 a_{1} d_{2}+11 b_{1} c_{2}=0,1024 a_{1}^{2} d_{1}+704 a_{1} b_{1} c_{1}+45 b_{1}^{3}=0$,

2.m) $m>3$. Then, we should have $b_{1}=0$ and, furthermore, either $c_{1}=c_{2}=0$ if $m$ is congruent with $1,2,4$ or 5 modulo 6 , or $d_{1}=d_{2}=0$ if $m$ is odd;

3. $a_{1}=\frac{4}{n(n+1)}$ with $n+1 / 2 \in \frac{1}{3} \mathbb{Z} \cup \frac{1}{4} \mathbb{Z} \cup \frac{1}{5} \mathbb{Z} \backslash \mathbb{Z}, b_{2}=0$ and either $c_{2}=0, b_{1}^{2}-3 a_{1} c_{1}=0$ or $c_{2} b_{1}-3 a_{1} d_{2}=0,2 b_{1}^{3}-9 a_{1} b_{1} c_{1}+27 a_{1}^{2} d_{1}=0$.

It is clear that the condition 1. in the above Theorem gives the Lamé and Hermite solutions (i), the condition 2.- the Brioschi-Halphen-Crowford solutions (ii), and the condition 3. - the Baldassarri solutions (iii). 


\section{References}

[1] Acosta-Humánez P, Blázquez-Sanz D, Non-integrability of some Hamiltonians with rational potentials, Discrete and Continuous Dynamical Systems Series B 2008; 10: 265293.

[2] Acosta-Humánez P. Galoisian Approach to Supersymmetric Quantum Mechanics. The integrability analysis of the Schrödinger equation by means of differential Galois theory, Dr Müller, Berlin: VDM Verlag; 2010.

[3] Acosta-Humánez P, Morales-Ruiz J, Weil J.-A. Galoisian Approach to integrability of Schrödinger Equation, Reports on Mathematical Physics 2011; 67(3): 305-374.

[4] Belmonte-Beitia J, Perez-Garcia VM, Vekslerchik V. Modulational instability, solitons and periodic waves in models of quantum degenerate boson-fermion mixtures. Chaos Solitons \& Fractals 2007; 32: 1268-77.

[5] Bludov Yu, Santhanam J, Kenkre VM, Konotop VV. Matter waves of Bose-Fermi mixtures in one-dimensional optical lattices. Phys Rev A 2006; 74: 043620, 14 pages.

[6] Duval A, Loday-Richaud M. Kovacic Algorithm and Its Application to Some Families of Special Functions, Appl Algebra Eng Commun Comput 1992; 3(3): 211-246.

[7] Guckenheimer J, Holmes P. Nonlinear Oscillations, Dynamical Systems, and Bifurcations of Vector Fields. New York: Springer; 1983.

[8] Karpiuk T, Brewczyk M, Ospelkaus-Schwarzer S, Bongs K, Gajda M, Rzazewski K. Soliton trains in Bose-Fermi mixtures. Phys Rev Lett 2004; 100410, 4 pages.

[9] Kostov N, Gerdjikov V, Valchev T. Exact Solutions for Equations of Bose-Fermi Mixtures in One-Dimensional Optical Lattice. SIGMA 2007; 3: 071, 14pp.

[10] Maciejewski A, Przybylska M. All meromorphically integrable 2D Hamiltonian systems with homogeneous potential of degree 3. Phys Lett A 2004; 327: 461-473.

[11] Morales-Ruiz J, Simó C. Non-integrability Criteria for Hamiltonians in the case of Lamé Normal Variational Equations, J Diff Eq 1996; 129: 111-135. 
[12] Morales-Ruiz J. Differential Galois Theory and Non integrability of Hamiltonian Systems, Prog. in Math., v. 179, Birkhäuser; 1999.

[13] Morales-Ruiz J, Ramis J-P, Simó C. Integrability of Hamiltonian systems and differential Galois groups of higher variational equations. Ann Scient Ec Norm Sup 2007; 40: 845-884.

[14] Morales-Ruiz J, Ramis J-P. Integrability of Dynamical systems through Differential Galois Theory: practical guide. Contemporary Math 2010; 509.

[15] Morales Ruiz J. A note on a connection between the Poincaré-Arnold-Melnikov integral and the Picard-Vessiot Theory. Banach Center Publications 2002; 58: 165-175.

[16] Perelomov A. Integrable Systems of Classical Mechanics and Lie Algebras. Vol. I. Basel: Birkhäuser; 1990.

[17] Przybylska M, Szumińsky W. Non-integrability of flail triple pendulum. Chaos, Solitons \& Fractals 2013; 53: 60-74.

[18] Rosemann S, Schöbel K. Open problems in the theory of finite-dimensional integrable systems and related fields. J Geom Phys 2015; 87: 396-414.

[19] Salerno M. Matter-wave quantum dots and antidots in ultracold atomic BoseFermi mixtures. Phys Rev A 2005; 72: 063602, 7 pages.

[20] van der Put M, Singer MF. Galois Theory of Linear Differential Equations. In: Grundlehren der Mathematischen Wissenschaften, vol. 328. Berlin: Springer; 2003.

[21] Wojciechowski S. Integrability of one particle in a perturbed central quartic potential. Physica Scripta 1985; 31: 433-438.

[22] Ziglin S. Self-intersection of the complex separatrices and the non-existence of integrals in the Hamiltonian systems with one-and-half degrees of freedom. J Appl Math Mech 1982; 45: 411-413. 